\title{
Diagnosis and Management of the Symptomatic Duodenal Diverticulum: a Case Series and a Short Review of the Literature
}

\author{
Beat Schnueriger • Stephan A. Vorburger • \\ Vanessa M. Banz • Alain M. Schoepfer • \\ Daniel Candinas
}

Received: 27 December 2007 / Accepted: 2 May 2008 /Published online: 3 June 2008

(C) 2008 The Society for Surgery of the Alimentary Tract

\begin{abstract}
Introduction The incidence of duodenal diverticula (DD) found at autopsy may be as high as $22 \%$. Perforation is the least frequent but also the most serious complication. This case series gives an overview of the management of this rare entity. Methods This study is a case series of eight patients treated for symptomatic DD.

Results Two patients had a perforated DD. One perforation was in segments III-IV, which to our knowledge is the first published case; the other perforation was in segment II. A segmental duodenectomy was performed in the first patient and a pylorus-preserving duodeno-pancreatectomy (pp-Whipple) in the second. A third patient with chronic complaints and recurring episodes of fever required an excision of the DD. In a fourth patient with biliary and pancreatic obstruction, a pp-Whipple was carried out, and a DD was discovered as the underlying cause. Four patients (one small perforation, one hemorrhage, and two recurrent cholangitis/pancreatitis caused by a DD) were treated conservatively.

Conclusions Symptomatic DD and, in particular, perforations are rare, encompass diagnostic challenges, and may require technically demanding surgical or endoscopic interventions. The diagnostic value of forward-looking gastroduodenoscopy in this setting seems limited. If duodenoscopy is performed at all, the use of a side-viewing endoscope is mandatory.

Keywords Duodenal diverticulum · Perforation ·

Operative management

\section{Introduction}

Duodenal diverticula (DD) were first reported by Chomel in $1710 .{ }^{1}$ The incidence of DD found at autopsy may be as

B. Schnueriger $(\bowtie) \cdot$ S. A. Vorburger $\cdot$ V. M. Banz $\cdot$ D. Candinas Department of Visceral and Transplantation Surgery,

University Hospital Bern,

CH-3010 Bern, Switzerland

e-mail: beat.schnueriger@insel.ch

A. M. Schoepfer

Department of Gastroenterology, Bern University Hospital, Bern, Switzerland

high as $22 \% .^{2}$ Similar incidences have been described during endoscopic retrograde cholangiopancreatography (ERCP). ${ }^{3,4}$

Most DD are asymptomatic; only 5\% of patients experience symptoms resulting from compression of neighboring organs, hemorrhage, or inflammation and perforation. ${ }^{5}$

Diverticula of the small intestine are largely pseudodiverticula, ${ }^{2,6,7}$ with the duodenum being the second most frequent location. ${ }^{8,9}$ These are typically located in the second portion of the duodenum within $2.5 \mathrm{~cm}$ of the ampulla of Vater. $^{8}$

Up until 2005, 115 cases of perforated DD have been published. $^{9-11}$ In $57 \%$ of all cases, the possible cause of duodenal perforation was peptic digestive processes as a result of the retention of food in the diverticula. ${ }^{12}$ Other causes, such as ulcerations, enterocoliths, blunt abdominal trauma, or iatrogenic perforation during an ERCP, have also been described. ${ }^{12-15}$
\end{abstract}


Here, we summarize the history of eight patients with symptomatic DD while reviewing the appropriate diagnostic steps and surgical therapy.

\section{Materials and Methods}

From January 2003 to December 2006, a total of eight patients with symptomatic DD were treated at our facility. Four patients required surgical intervention, and four patients were treated conservatively. Prehospitalization data, inpatient chart records, and radiological and endoscopic findings were collected and analyzed retrospectively. We then compared our results with the existing literature.

\section{Results}

Case Report \#1 A 68-year-old man was admitted with acute abdominal pain accompanied by nausea and bilious vomiting, which he had experienced for $6 \mathrm{~h}$. The patient's personal history included Crohn's disease, which was in complete clinical remission under daily therapy with $150 \mathrm{mg}$ of azathioprine. The clinical examination showed a patient with diffuse pain in the lower abdomen radiating to both flanks as well as sparse bowel sounds. Blood analysis showed an elevated white blood cell count with $15 \mathrm{~g} / 1$ and a normal Creactive protein (CRP) level. The result of an abdominal radiography was also normal. Twelve hours after admission, the CRP level increased to $210 \mathrm{mg} / 1$ (normal $<4 \mathrm{mg} / \mathrm{l})$. The abdominal computed tomography (CT) scan is shown in Fig. 1. A forward-looking gastroduodenoscopy was carried out in order to locate the site of perforation. The examination revealed macroscopically normal mucosa up to $40 \mathrm{~cm}$ past the ligament of Treitz, without detecting any perforation site. In this setting, we opted for laparotomy and surgical revision. After mobilization of the duodenum (Kocher maneuver), pus was found dorsal to duodenal segment III/IV, coming up from behind the pancreas. Further examination showed a macerated 5-cm-large DD in segment IV with multiple perforation sites (Figs. 2 and 3). This was accompanied by a retroperitoneal phlegmona that extended to the left pararenal region. The pancreas itself was normal. In this setting, we carried out a pancreas-preserving duodenectomy of segments III and IV with an end-to-end duodeno-jejunostomy (Fig. 4) $3 \mathrm{~cm}$ distal of the ampulla of Vater. The azathioprine treatment was paused so that the patient could be treated for 5 days with imipenem. After 15 days, the patient was dismissed from hospital in good health.

Case Report \#2 A 70-year-old woman presented with epigastric pain that was ongoing for $4 \mathrm{~h}$ and radiating to the right hemiabdomen. Nausea was followed by bilious

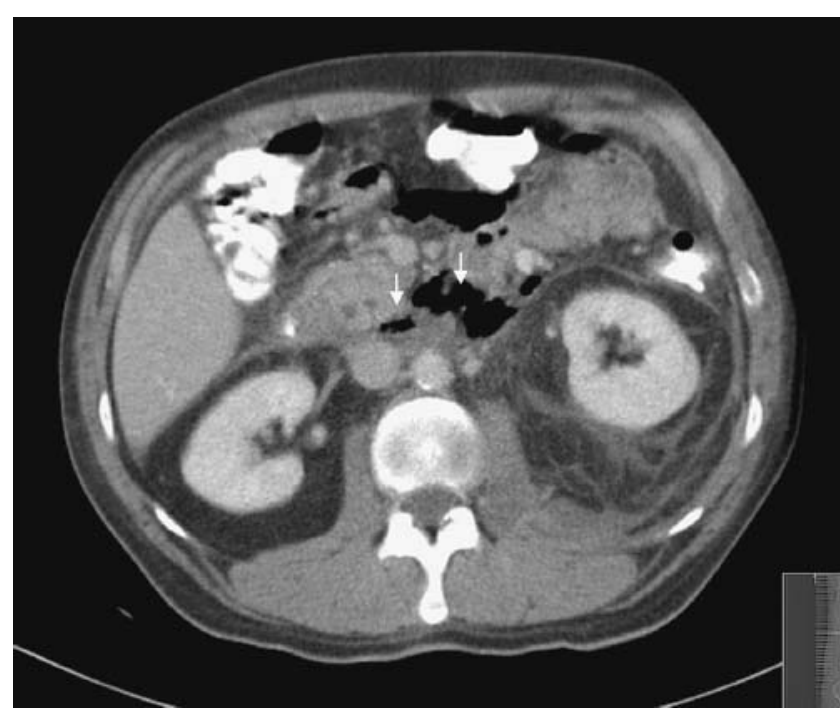

Figure 1 Abdominal CT scan of a 68-year-old man with a perforated duodenal diverticulum. The scan shows extraluminal retroperitoneal air around the ascending duodenal segment (segment IV) (white arrows) as well as minimal retroperitoneal, pararenal fluid with an impressive fat stranding in a 68 -year-old male patient with a perforated duodenal diverticulum.

vomiting. The clinical examination revealed peritonitis in the right upper quadrant. Her white blood cell count was elevated $(16.8 \mathrm{~g} / \mathrm{l})$, whereas the CRP level was normal. Result of a plain abdominal X-ray was normal. Due to persistent abdominal pain, we carried out a CT scan which showed a retroperitoneally perforated DD in segments IIIII. Because of her increasing abdominal discomfort, we performed an emergency laparotomy. Complete duodenal mobilization revealed a very firm pancreatic head. Intraoperative sonography showed a nondilated pancreatic duct and homogenous pancreatic parenchyma. An intraoperative gastroduodenoscopy was carried out, allowing for maximal diagnostic security in order to rule out further pathologies. Intraoperative gastroduodenoscopy failed to locate the DD. Due to the ambiguous findings in the pancreatic head, we carried out a pp-Whipple. A DD, covered in pus and fibrinous strands, was located between segments II and III of the duodenum. Dissection of the $5 \times 5$-cm-large diverticulum showed an opening of $7 \mathrm{~mm}$ towards the duodenal mucosa that was filled with partially digested food. The patient's further recovery was uneventful.

Case Report \#3 A 68-year-old man presented to his physician with a feeling of general illness, nonspecific upper abdominal pain, as well as vomiting and diarrhea which had been ongoing for several days. A colonoscopy showed no pathology. Due to persistent epigastric abdominal pain, intermittent lack of appetite, and a dislike for meat, a gastroduodenoscopy was performed. The examination revealed a grade II reflux esophagitis with a small axial hernia and minimal antrum gastritis. Besides these findings, 


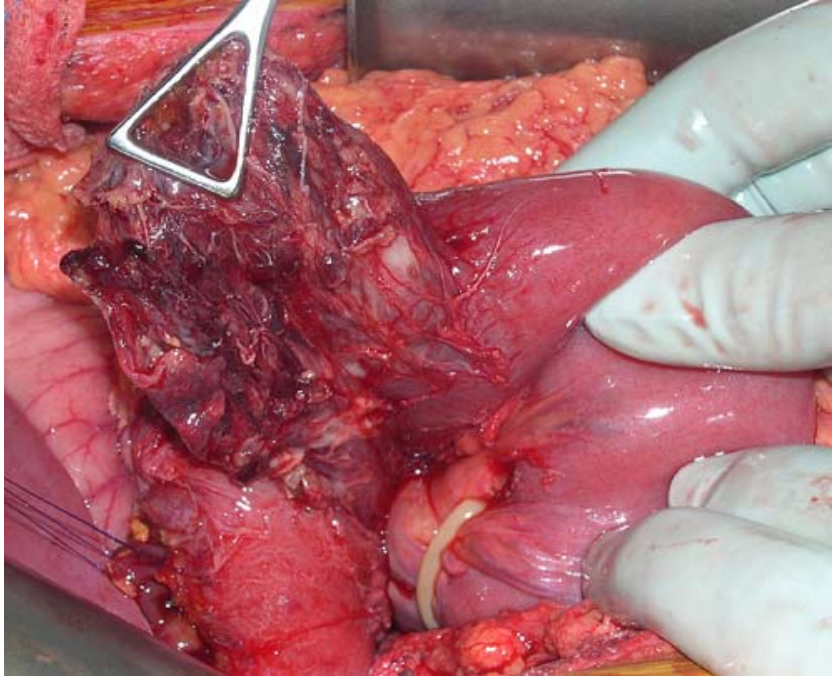

Figure 2 Macerated 5-cm-large duodenal diverticulum in segment IV with multiple perforation sites.

no further pathologies were detected. In the course of time, the patient developed recurrent fever. A CT scan of the abdomen revealed a heterogeneous zone in the transition area between the head and the neck of the pancreas. Endosonography confirmed this finding, but needle biopsy was inconclusive. The patient was referred to our clinic. At the time of examination, the patient was in good general health without abdominal pain. A magnetic resonance imaging (MRI) of the abdomen showed a heterogeneous zone of the pancreatic body without obstruction of the surrounding structures. As malignancy could not be ruled out with certainty, we opted for surgical exploration. The intraoperative aspect revealed a normal pancreas. Sonography and multiple biopsies were without pathology. As a possible cause for the recurrent fever, we found a DD

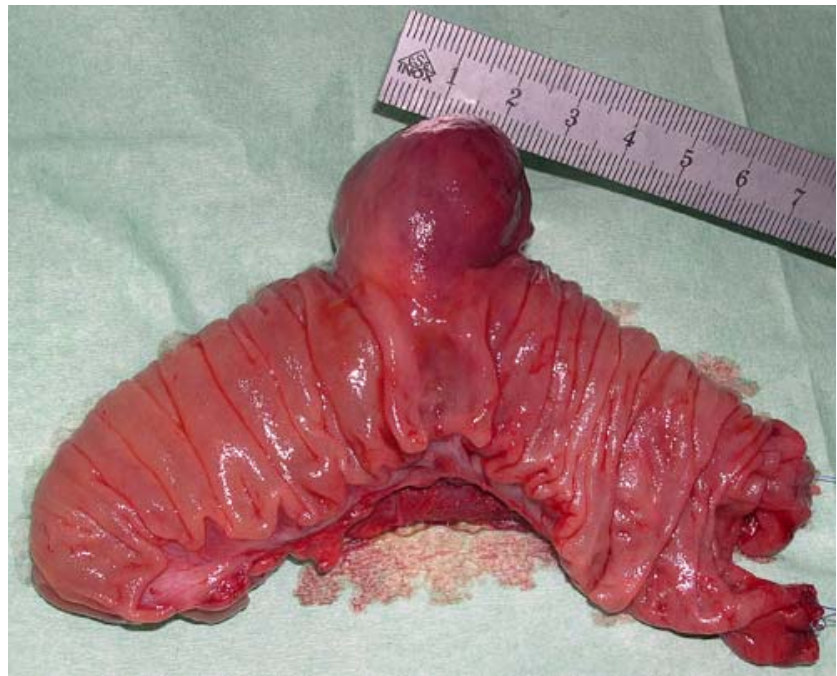

Figure 3 Resected segments III and IV duodena everted duodenal diverticulum.

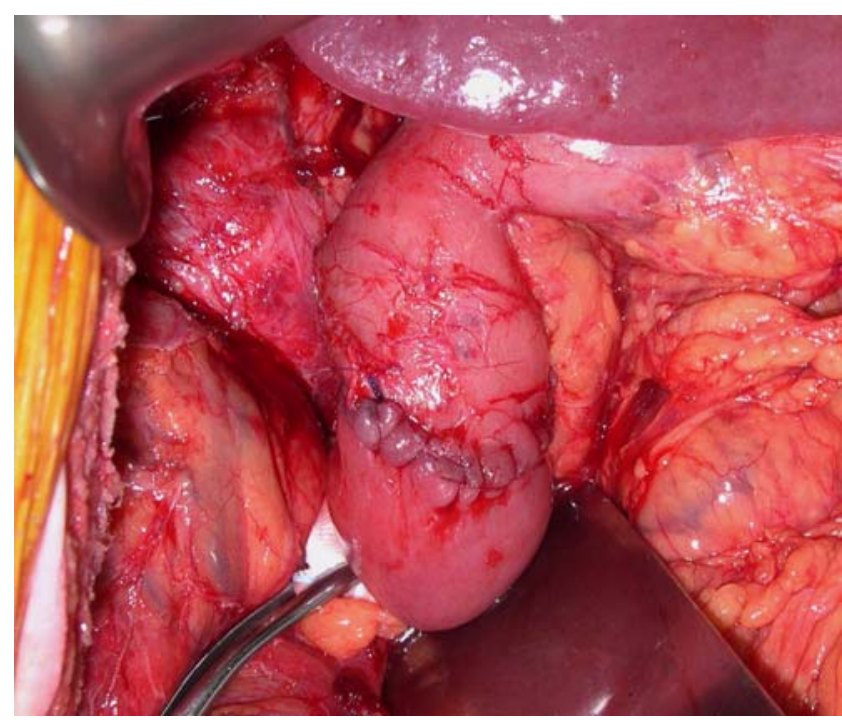

Figure 4 Hand-sewn end-to-end duodeno-jejunostomy after segmental duodenectomy of segments III and IV.

located in segment II. The diverticulum was excised and the duodenum repaired with full-thickness sutures. Due to its proximity to the ampulla of Vater, we carried out a biliodigestive anastomosis according to Roux-en-Y. Definitive histological examination confirmed the duodenal pseudodiverticulum without acute inflammation. Postoperative recovery was uneventful, and the patient was dismissed on the 12th postoperative day.

Case Report \#4 A routine medical checkup of a 65-yearold man with no complaints revealed slightly elevated blood amylase and lipase values. Six months later, an ERCP was performed because the elevated values had persisted. The ERCP showed a significant stenosis of the Wirsungian duct, 3-4 cm proximal to the ampulla of Vater. The orifice of a DD was located immediately proximal to the ampulla. A CT of the abdomen failed to clearly identify the boundaries of the head of the pancreas, with a small bubble of air visible in direct proximity to the pancreatic duct. A cholangio-MRI revealed a dilated pancreatic duct of $1 \mathrm{~cm}$ right up to the ampulla of Vater. The results were discussed with the patient, and the necessity for explorative laparotomy was agreed upon, as a tumor could not be ruled out with certainty. Furthermore, chronic outlet obstruction of the pancreatic duct may have led to secondary exocrine and endocrine problems with the pancreas.

Intraoperative findings revealed a markedly enlarged pancreatic head and uncinate process. Gradual resection of the pancreatic head revealed a 3-4-cm-large periampullary DD with compression of the pancreatic duct. In such a setting, a pp-Whipple operation was carried out. Definitive histological examination confirmed the presence of a periampullary duodenal pseudodiverticulum with a narrow opening and minimal chronic-atrophic partially fibrosing 
pancreatitis. The patient was dismissed without complications on the 11th postoperative day. No signs of endocrine or exocrine pancreatic insufficiency were found upon follow-up.

Of the four patients not requiring surgery, patient 5 had a DD located in duodenal segment III with a small iatrogenic perforation, which occurred during an ERCP because of biliary obstruction. We treated this patient conservatively by a temporary percutaneous transhepatic cholangio drainage (PTCD) inserted under CT guidance and a course of antibiotics and parenteral nutrition.

Patient 6 had a hemodynamically relevant hemorrhage from a DD due to coagulopathy in a Child C hepatopathy. The bleeding and the DD were diagnosed by ERCP, but a treatment by endoscopic clipping or sclerotherapy was not possible. Finally, the bleeding stopped after correction of the coagulopathy by the substitution with fresh frozen plasma.

Patients 7 and 8 suffered from cholangitis caused by an infected DD. Both received an ERCP with papillotomy with insertion of a temporary naso-biliary drainage and a treatment with antibiotics. Table 1 gives an overview of our eight patients with symptomatic DD.

\section{Discussion}

This case series shows a wide spectrum of diagnostic and therapeutic problems DD can give. To the best of our knowledge and after an extensive search of the literature, we report here the first patient with perforated DD in duodenal segments III-IV.

Clinical Symptoms and the Diagnosis of Complicated $D D$ Clinical symptoms are usually nonspecific. In the case of a perforation, patients often experience a per-acute onset of pain followed by nausea and vomiting. Furthermore, chronic progression with pain and fever due to recurrent episodes of cholangitis or pancreatitis or by the inflammation of the DD itself are possible symptoms, as was also seen in our patients $3,4,7$, and 8 . Additionally, anorexia and steatorrhoe due to duodeno-colic fistulas have also been described. ${ }^{16}$

Correctly diagnosing the complications associated with DD, especially duodenal perforation, poses several difficulties. Fifty percent of all conventional radiological examinations show no abnormalities. ${ }^{10}$ An abdominal CT scan is the most sensitive examination if there is suspicion that a DD perforation may have occurred. Findings consist of a thickened bowel wall, mesenteric fat stranding, and an extraluminal, retroperitoneal collection of air or fluid. ${ }^{17,18}$ Occasionally, a contrast-enhanced CT scan can directly depict a DD. ${ }^{13}$ Duarte et al. and Juler et al. reported 101 cases of duodenal perforation, of which only 13 were diagnosed preoperatively. ${ }^{12,19}$ Because there are no pathognomonic signs, even making the correct intraoperative diagnosis requires a high index of suspicion.

The Value of Endoscopy The diagnostic value of the forward-viewing gastroduodenoscopy remains doubtful, as was seen in three of our four surgically managed patients, where repeated gastroduodenoscopies failed to reveal any pathology. This can be explained by the difficulty of the forward-viewing endoscope to reliably assess the concavity of duodenal segment II retroperitoneal in the vicinity of the ampulla of Vater where most $(75 \%)$ of the DD are located. ${ }^{11}$ Additionally, an acute diverticulitis causes mucosal swelling and narrowing of the diverticular orifice that further hampers the diagnostic yield of forward upper endoscopy. Our experience shows that forward-viewing

Table 1 Overview of All Patients with Symptomatic DD

\begin{tabular}{|c|c|c|c|}
\hline Patient & Location of DD & Complication & Treatment \\
\hline 1 & Segments III-IV & Acute retroperitoneal perforation & Segmental duodenectomy \\
\hline 2 & Segment III & Acute retroperitoneal perforation & $\begin{array}{l}\text { Pylorus-preserving duodeno-pancreatectomy } \\
\text { (pp-Whipple) }\end{array}$ \\
\hline 3 & Segment II & Chronic complaints and recurring episodes of fever & Excision of the diverticula \\
\hline 4 & Segment II & $\begin{array}{l}\text { Chronic biliary and pancreatic obstruction } \\
\text { with chronic-atrophic pancreatitis }\end{array}$ & $\begin{array}{l}\text { Pylorus-preserving duodeno-pancreatectomy } \\
\text { (pp-Whipple) }\end{array}$ \\
\hline 5 & Segment III & $\begin{array}{l}\text { Small iatrogenic perforation caused by an } \\
\text { ERCP (biliary obstruction) }\end{array}$ & PTCD, period of parenteral nutrition and antibiotics \\
\hline 6 & Segment II & Hemorrhage (Child C hepatopathy) & Conservative, fresh frozen plasma \\
\hline 7 & Segment II & Infection and biliary obstruction & $\begin{array}{l}\text { ERCP with papillotomy and insertion of a } \\
\text { naso-biliary tube, antibiotics }\end{array}$ \\
\hline 8 & Segment II & $\begin{array}{l}\text { DD infection with biliary obstruction } \\
\text { and cholangitis }\end{array}$ & $\begin{array}{l}\text { ERCP with papillotomy and insertion of an } \\
\text { naso-biliary tube, antibiotics }\end{array}$ \\
\hline
\end{tabular}

$D D$ Duodenal diverticulum, $P T C D$ percutaneous transhepatic cholangio drainage, $E R C P$ endoscopic retrograde cholangiopancreatography 
endoscopy can exclude other pathologies, such as ulcers, but has a low negative predictive value in diagnosing DD. A side-viewing duodenoscope (as was used in our fourth to eighth patients) may be of benefit and allow for a correct diagnosis as shown previously by Leivonen et al., ${ }^{3}$ who demonstrated that, in 1,735 ERCPs, DD were found in 123 patients $(7.1 \%)$. Jin et al. found DD in 129 of 527 patients (24.6\%) upon ERCP. ${ }^{4}$

ERCP not only has its own diagnostic value but may provide endoscopic therapy options in some patients with symptomatic DD. Up until 2006, there were nine reported cases of endoscopic treatment of hemorrhage from a DD and one documented endoscopically drained retroperitoneal abscess which occurred after DD perforation. ${ }^{20,}{ }^{21}$ Lee et al. presented another 11 patients with endoscopic treatment of symptomatic DD (obstruction, pain, pancreatitis). ${ }^{22}$ Several other unusual case reports can be found, including a bezoar in a periampullary DD causing pancreatitis ${ }^{23}$ or a vegetable stalk as a nidus for gallstone formation in a DD. ${ }^{24}$ Both could be removed during ERCP.

Surgical Treatment Surgical intervention is usually only required when there are complications, whereof perforation is the least frequent but also one of the most serious complications. ${ }^{25}$ However, nonoperative or endoscopic treatment of perforations has also been described. ${ }^{20}$ An additional example is our fifth patient. However, care must be taken not to delay the surgical treatment of a perforated $\mathrm{DD}$, as this condition is associated with a mortality of up to $13 \% .{ }^{12}$ More often than perforation are symptoms from the pancreaticobiliary system, such as recurrent cholangitis or pancreatitis, as a result of increased pressure in a poorly emptying and inflammed DD. ${ }^{25}$ Harthun et al. published another unusual indication for surgery: a duodenal obstruction caused by an intraluminal DD, which was resected through a longitudinal duodenotomy. ${ }^{26}$

Concerning surgical techniques and options, detailed recommendations or even guidelines are lacking. Because of the rare appearance of symptomatic DD, only case reports or case series up to four patients have been published until now. In case of perforation, surgical options range from local excision of the diverticulum to a pp-Whipple, depending on the site of the DD and the grade of inflammation. Most frequently, resection of the DD after Kocher maneuver with one- or two-layer closure of the duodenum has been described in this context. ${ }^{7,18,27}$ As a third option, we describe here a segmental duodenal resection of a perforated DD in segments III-IV. This option can only be considered in the rare case of a DD located in segments III and IV. A ppWhipple may be necessary when the sac of the DD lies in close proximity to the common bile and pancreatic ducts, and quite frequently the ampulla of Vater is found within the diverticulum. ${ }^{28}$ Either a pp-Whipple or a segmental duodenal resection with removal of the nectrotic tissue must be considered in case of perforation which leads to severe retroperitoneal inflammation.

If the leading symptoms of DD are obstruction of the biliary tract (cholangitis) or the pancreatic duct (pancreatitis), a resection of the duodenum is not required. In the absence of other risk factors and the presence of a DD, exclusion of the duodenum by means of Roux-Ycholedochojejunostomy (biliodigestive anastomosis) and duodeno-jejunostomy has warranted satisfactory results. ${ }^{29,30}$

All the above procedures are technically challenging and carry the risk of potential injury to the pancreatic duct and parenchyma as well as to the extrahepatic bile ducts. Other potential complications are duodenal fistulas, sepsis, intraabdominal abscesses, and pancreatitis.

\section{Conclusion}

Complications caused by DD and, in particular, perforations are rare and encompass diagnostic challenges requiring immediate and usually technically demanding surgical interventions. A high index of suspicion is required for the correct diagnosis. In our opinion, if the presence of a DD seems likely, the side-viewing duodenoscope should be preferred to the forward-viewing endoscope for higher diagnostic yield.

\section{References}

1. Chomel J. Report of a case of duodenal diverticulum containing gallstones. Acad R Sci Paris 1710:48-50.

2. Ackermann W. Diverticula and variations of the duodenum. Ann Surg 1943;117:403-413.

3. Leivonen MK, Halttunen JA, Kivilaakso EO. Duodenal diverticulum at endoscopic retrograde cholangiopancreatography, analysis of 123 patients. Hepatogastroenterology 1996;43:961-966.

4. Jin T, Ho G, Eun Y, Joong G, Chang H, Young S et al. Incidence of juxtapapillary duodenal diverticula is higher in brown pigment stone but not in black pigment or cholesterol stone. Gastroint Endosc 2006;63:297.

5. Oddo F, Chevallier P, Souci J, Baque J, Buckley MJ, Fabiani P et al. Radiologic aspects of the complications of duodenal diverticula. J Radiol 1999;80(2):134-140.

6. Suda K, Mizuguchi K, Matsumoto M. A histopathological study on the etiology of duodenal diverticulum related to the fusion of the pancreatic anlage. Am J Gastroenterol 1983;78(6):335-338.

7. Yang HK, Fondacaro PF. Enterolith ileus: a rare complication of duodenal diverticula. Am J Gastroenterol 1992;87(12):18461848.

8. Chitambar IA, Springs C. Duodenal diverticula. Surgery 1953;33 (5):768-791.

9. Umbricht-Sprungli RE, Hollinger A, Meier L, Largiader F. Complications of diverticuli of the proximal small intestine. 3 case reports and review of the literature. Chirurg 1992;63(7): $568-571$. 
10. Papalambros E, Felekouras E, Sigala F, Kiriakopoulos A, Giannopoulos A, Aessopos A et al. Retroperitoneal perforation of a duodenal diverticulum with colonic necrosis-report of a case. Zentralbl Chir 2005;130(3):270-273.

11. Jones TW, Merendino KA. The perplexing duodenal diverticulum. Surgery 1960;48:1068-1084.

12. Duarte B, Nagy KK, Cintron J. Perforated duodenal diverticulum. Br J Surg 1992;79(9):877-881.

13. Franzen D, Gurtler T, Metzger U. Solitary duodenal diverticulum with enterolith as a rare cause of acute abdomen. Swiss Surg 2002;8(6):277-279.

14. Poostizadeh A, Gow KW, Al-Mahmeed T, Allardyce DB. Traumatic perforation of duodenal diverticulum. J Trauma 1997;43(2):370-371.

15. Elder J, Stevenson G. Delayed perforation of a duodenal diverticulum by a biliary endoprosthesis. Can Assoc Radiol J 1993;44(1):45-48.

16. Tissot E, Ayoun CL, Maisonnier M. Preoperative diagnosis of a subacute perforation of a duodenal diverticulum. J Chir (Paris) 1988;125(5):346-349.

17. Van Beers B, Trigaux JP, De Ronde T, Melange M. CT findings of perforated duodenal diverticulitis. J Comput Assist Tomogr 1989;13(3):528-530.

18. Cavanagh JE Jr. Iatrogenic perforation of perivaterian duodenal diverticulum: report of a case. Can J Surg 1996;39(4):336-338.

19. Juler GL, List JW, Stemmer EA, Connolly JE. Perforating duodenal diverticulitis. Arch Surg 1969;99(5):572-578.

20. Eeckhout G, Vanstiphout J, Van Pottelbergh I, Leyman P, Vandervoort J, De Man M et al. Endoscopic treatment of a perforated duodenal diverticulum. Endoscopy 2000;32(12):991-993.

21. Yoshino $\mathrm{S}$ et al. Endoscopic management of a dieulafoy-like lesion in a duodenal diverticulum. Dig Endosc 2006;18:59-61.
22. Lee SH, Park SH, Lee JH, Park DH, Chung IK, Kim SJ et al. Endoscopic diverticulotomy with an isolated-tip papillotome (Iso-Tome) in a patient with intraluminal duodenal diverticulum. Gastrointest Endosc 2005;62(5):817-819.

23. Pothamsetty S, Attar B, Abrahamian F, Behara S, Rahim T. Bezoar in a periampullary extraluminal duodenal diverticulum causing acute pancreatitis. Am J Gastroenterol 2000;95:728.

24. Plath F, Brock P, Hasse N, Liebe S, Arendt T. Vegetable stalk as a nidus for gallstone formation in a patient with a juxtapapillary duodenal diverticulum. Gastrointest Endosc 2002;56 (6):944-946.

25. Lobo DN, Balfour TW, Iftikhar SY, Rowlands BJ. Periampullary diverticula and pancreaticobiliary disease. Br J Surg 1999;86 (5):588-597.

26. Harthun NL, Morse JH, Shaffer HA Jr, Minasi JS. Duodenal obstruction caused by intraluminal duodenal diverticulum and annular pancreas in an adult. Gastrointest Endosc 2002;55 (7):940-943.

27. Yokomuro S, Uchida E, Arima Y, Mizuguchi Y, Shimizu T, Kawahigashi Y et al. Simple closure of a perforated duodenal diverticulum: "a case report". J Nippon Med Sch 2004;71(5): 337-339.

28. Willox GL, Costopoulos LB. Entry of common bile and pancreatic ducts into a duodenal diverticulum. Cause of pancreaticobiliary disease. Arch Surg 1969;98(4):447-450.

29. Vassilakis JS, Tzovaras G, Chrysos E, Mouzas I, Manousos O, Xynos E. Roux-Y choledochojejunostomy and duodenojejunostomy for the complicated duodenal diverticulum. Am J Surg 1997;174(1):45-48.

30. Critchlow JF, Shapiro ME, Silen W. Duodenojejunostomy for the pancreaticobiliary complications of duodenal diverticulum. Ann Surg 1985;202(1):56-58. 